\title{
FTIR Spectroscopy Combined with Partial Least Square for Analysis of Red Fruit Oil in Ternary Mixture System
}

\author{
A. Rohman, Dwi Larasati Setyaningrum, and Sugeng Riyanto \\ Laboratory of Analytical Chemistry, Department of Pharmaceutical Chemistry, Faculty of Pharmacy, \\ Gadjah Mada University, Yogyakarta 55281, Indonesia \\ Correspondence should be addressed to A. Rohman; abdulkimfar@gmail.com
}

Received 26 February 2014; Accepted 8 May 2014; Published 21 May 2014

Academic Editor: Kam-Sing Wong

Copyright (C) 2014 A. Rohman et al. This is an open access article distributed under the Creative Commons Attribution License, which permits unrestricted use, distribution, and reproduction in any medium, provided the original work is properly cited.

FTIR spectroscopy is a promising method for quantification of edible oils. Three edible oils, namely, red fruit oil (RFO), corn oil (CO), and soybean oil (SO), in ternary mixture system were quantitatively analyzed using FTIR spectroscopy in combination with partial least square (PLS). FTIR spectra of edible oils in ternary mixture were subjected to several treatments including normal spectra and their derivative. Using PLS calibration, the first derivative FTIR spectra can be exploited for determination of RFO; meanwhile, the second derivative spectra were preferred for determination of CO and SO. The $R^{2}$ values obtained for the relationship between actual and FTIR predicted values of RFO, CO, and SO were $0.9863,0.9276$, and 0.9693 , respectively. The root mean square error of calibration (RMSEC) values obtained were 1.59, 1.72, and 1.60\% (v/v) for RFO, CO, and SO, respectively. The result showed that FTIR spectroscopy offers accurate and reliable technique for quantitative analysis of RFO, CO, and SO simultaneously in ternary mixture systems. Besides, the developed method can be extended for analysis of $\mathrm{CO}$ and $\mathrm{SO}$ as adulterants in RFO.

\section{Introduction}

In the fats and oils industry, red fruit oil (RFO) has become an emerging functional oil, especially in Indonesia. RFO is obtained from the extraction of red fruit (Pandanus conoideus Lam.). The extract of red fruit has been reported to possess antioxidant activities [1]. For local community in Papua province of Indonesia as well as Papua New Guinea, RFO is believed to be preventive agents of several degenerative diseases such as cancer, atherosclerosis, and coronary heart disease.

Compared with common vegetable oils available in the market, such as corn and soybean oils, RFO is more expensive by $10-15$ times. Consequently, RFO can be easily faked with other cheaper oils like corn oil (CO) and soybean oil (SO) in order to get the maximum profits. Even though RFO's adulteration does not cause health problems, the primary consumer rights are violated by the fraudulent practices [2, 3]. The practice of adulteration can involve the dilution of
RFOs with one or more oils. Therefore, the detection and quantification of oil adulterants such as $\mathrm{CO}$ and $\mathrm{SO}$ in $\mathrm{RFO}$ are highly demanded.

Edible fats and oils are usually analyzed by determining certain components of fats and oils such as fatty acids using gas liquid chromatography and triglyceride composition using reversed phase HPLC, rather than analysis of fats and oils as a whole matter. As one of the vibrational spectroscopy methods, Fourier transform infrared (FTIR) spectroscopy can be developed for analysis of fats and oils as a whole matter in order to overcome this problem [4]. FTIR spectroscopy has been accepted and widely used in food research. This technique has emerged as a powerful analytical tool in qualitative and quantitative analysis of specific components present in food, especially in combination with certain chemometrics package [5].

FTIR spectroscopy is fast, sensitive, and nondestructive, with minimum sample preparation [6]. Combined with multivariate calibrations, FTIR spectroscopy can provide 
a powerful technique to detect oil adulterants in high priced oils including RFO. Currently, FTIR spectroscopy coupled with user friendly sampling technique of attenuated total reflectance (ATR) has been an emerging technique for analysis of edible fats and oils due to its capability as "fingerprint technique," either for qualitative or for quantitative analysis $[7,8]$. Analysis of fats and oils using FTIR spectroscopy can also be considered as "green analytical chemistry" because this technique reduces chemical reagents which are hazardous to human health and environment [9].

From the several multivariate calibration techniques, partial least square (PLS) has a large number of applications for quantitative analysis in the complex mixtures. PLS allows sophisticated statistical approach to use the full spectral regions rather than using a specific region. Besides, PLS needs a calibration step where the relation between the spectra and the analyte concentrations is deduced from a set of reference samples [10]. With PLS, it is possible to make a calibration for the desired component while completely modeling the other source of variations [11].

FTIR spectroscopy in combination with multivariate calibrations has been reported for quantitative analysis of certain oils for authentication study such as canola, walnut, sunflower, peanut, and sesame oils in olive oil [12], hazelnut oil in extra virgin olive oil (EVOO) [13], palm oil in sesame oil [14], lard in cod-liver oil [15], palm kernel oil in virgin coconut oil [16], and canola oil and rice bran oil in red fruit oil [17]. In this study, we developed FTIR spectroscopy in combination with PLS calibration for simultaneous analysis of RFO mixed with soybean oil (SO) and corn oil (CO) in ternary mixture system.

\section{Materials and Methods}

Red fruit was taken from Papua, Indonesia. Botanical identification of red fruit was performed by the Department of Pharmaceutical Biology, Faculty of Pharmacy, Gadjah Mada University, Jogjakarta, Indonesia. Corn oil (CO) and soybean oil (SO) were purchased from the local market in Jogjakarta, Indonesia.

2.1. Calibration and Validation. For calibration model, 44 samples composed of RFO, CO, and SO in ternary mixture system with certain concentrations were prepared. For validation set, 26 independent samples which were different from calibration samples containing RFO, CO, and SO in the concentration range of $0-100.0 \%$ were also prepared. Each sample either in calibration or validation sets was subjected for FTIR analysis.

2.2. FTIR Spectra Measurements. A FTIR spectrophotometer of ABB3000 fitted with a preserved and desiccated interferometer was used for FTIR spectra acquisition. The detector and beam splitter used are deuterated triglycine sulphate (DTGS) and potassium bromide (KBr), respectively. The sampling compartment was horizontal attenuated total reflectance (HATR). Approximately $1.0 \mathrm{~mL}$ of oil was subjected to HATR composed of zinc selenide ( $\mathrm{ZnSe}$ ) crystal.
All spectral measurements were acquired over midinfrared region (4000-650 $\mathrm{cm}^{-1}$ ) using 32 scanning at resolution of $8 \mathrm{~cm}^{-1}$. For quantitative analysis, single-beam ATR spectra were collected from each sample and read as absorbance units. As a background, an air spectrum was used. Spectra were collected in duplicate and averaged before being used for model optimization.

2.3. Statistical Analysis. Multivariate calibration of partial least square (PLS) was performed using the software Horizon MB (Canada), employing the original spectra and first and second derivative-transformed spectra. The values of coefficient of determination $\left(R^{2}\right)$ and root mean square error of calibration (RMSEC) were used as performance criteria for calibration model. Consider the following:

$$
\text { RMSEC }=\sqrt{\frac{\sum_{i=1}^{n}(\text { actual }- \text { calculated })^{2}}{N-f-1}} .
$$

RMSEC refers to the uncertainty of calibration for the selected ternary system; a small value of RMSEC indicates that the calibration has less error. Furthermore, the predictive ability of the model was assessed using $R^{2}$ values and root mean square error of prediction (RMSEP) which indicates how well the developed model will perform the quantitative analysis of new samples; the lower the RMSEP value, the better the prediction power of developed calibration model

$$
\text { RMSEP }=\sqrt{\frac{\sum_{i=1}^{m}(\text { actual }- \text { calculated })^{2}}{M-1}} .
$$

The term "actual" refers to the known or true concentration of selected oils in the ternary mixtures; meanwhile, the term "calculated" refers to a value computed by the model using spectral data; $N$ and $M$ are the number of samples used in the calibration and validation sets, respectively; $f$ is the number of factors used in the calibration model [18].

2.4. Fatty Acids Analysis. Fatty acid composition of studied vegetable oils (red fruit oil, corn oil, and soybean oil) was determined using gas liquid chromatography with flame ionization detection (GC-FID) as derivative of fatty acid methyl ester (FAME), according to previously reported studies by Gurdeniz and Ozen [19]. Quantification of each fatty acid was based on internal normalization area.

\section{Results and Discussion}

3.1. Spectral Analysis. Triglycerides are the main components of edible fats and oils; as a consequence, FTIR spectra of triglycerides dominate in FTIR spectra of fats and oils [20]. The overlay of FTIR spectra of red fruit oil (RFO), corn oil (CO), and soybean oil (SO) at midinfrared region (4000$650 \mathrm{~cm}^{-1}$ ) is presented in Figure 1. Each peak corresponds to functional groups responsible for infrared absorption; meanwhile, the intensities of each peak were correlated with the concentration of functional groups present in analytes (RFO-CO-SO). 


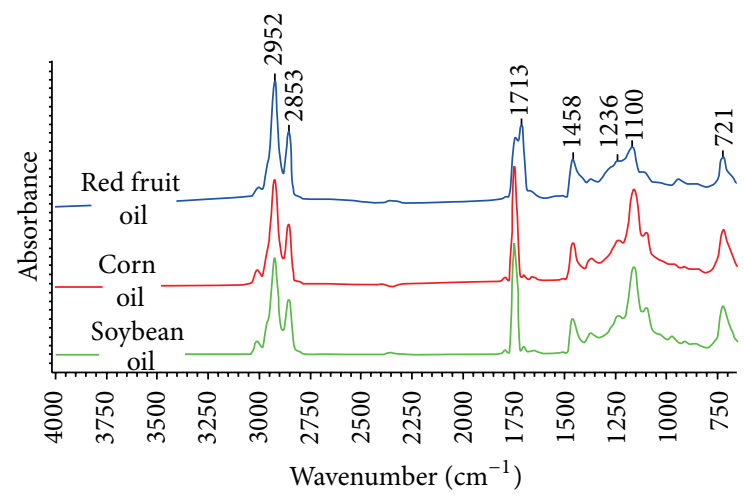

FIGURE 1: FTIR spectra of studied edible oils which are scanned at midinfrared region $\left(4000-650 \mathrm{~cm}^{-1}\right)$.

The FTIR spectra of evaluated vegetable oils in Figure 1 look very similar; however, they reveal slight differences in their spectra which can be observed in region around $1700-1780 \mathrm{~cm}^{-1}$ and at fingerprint region $\left(1500-650 \mathrm{~cm}^{-1}\right)$. This is not surprising because FTIR spectra are taken into account as fingerprint technique meaning that two different oils shall have different FTIR spectra. There is no band at $1712 \mathrm{~cm}^{-1}$ for CO and SO, while RFO has peak at $1712 \mathrm{~cm}^{-1}$ due to $\mathrm{C}=\mathrm{O}$ stretching vibration. Furthermore, at frequency region of $910-960 \mathrm{~cm}^{-1}$, these oils also revealed different peak intensities due to the difference of fatty acids composition. Fatty acid composition of RFO, CO, and SO was compiled in Table 1. Analysis of fatty acids revealed that the main component of RFO is oleic acid; meanwhile, $\mathrm{CO}$ and $\mathrm{SO}$ contain linoleic acid as their main component.

\subsection{Selection of Spectra Regions for Quantitative Analysis.} The quantitative analysis of RFO, CO, and SO in ternary mixtures was carried out using multivariate calibration of PLS algorithm. In PLS model, evaluation of the method linearity was performed in order to show the proportional relationship between responses (absorbances) versus analyte concentrations of RFO, CO, and SO. The samples of edible oils were independently separated into calibration and validation data sets in order to get similar standard deviation and average values, so that both data sets spanned the working range of the studied edible oils [11].

The optimization process for determination of RFO, CO, and $\mathrm{SO}$ in ternary mixture systems was initially performed by selecting the frequency regions capable of describing most characteristics of oil components to be determined. After being selected, the spectral regions were further used for developing PLS calibration model. PLS can be applied for the analysis of the component of interest using whole FTIR spectral regions rather than specific regions [9]. For this reason, some frequency regions were evaluated in terms of their capability to provide the highest correlation between actual and FTIR predicted values using PLS calibration. Table 2 revealed values of coefficients of determination $\left(R^{2}\right)$ using optimized frequency regions. Regions that exhibit
TABLE 1: Fatty acid composition of red fruit oil (RFO), corn oil (CO), and soybean oil (SO).

\begin{tabular}{lccc}
\hline Fatty acids & RFO & CO & SO \\
\hline$(\mathrm{C} 14: 0)$ & $0.08 \pm 0.01$ & $0.12 \pm 0.00$ & $0.01 \pm 0.00$ \\
$(\mathrm{C} 16: 0)$ & $17.48 \pm 0.07$ & $10.15 \pm 0.62$ & $10.97 \pm 0.12$ \\
$(\mathrm{C} 18: 0)$ & $\mathrm{ND}^{*}$ & $2.42 \pm 0.08$ & $5.10 \pm 0.16$ \\
$(\mathrm{C} 18: 1)$ & $79.53 \pm 0.13$ & $26.24 \pm 0.72$ & $20.75 \pm 0.52$ \\
$(\mathrm{C} 18: 2)$ & $1.56 \pm 0.14$ & $58.60 \pm 1.05$ & $52.24 \pm 0.86$ \\
$(\mathrm{C} 18: 3)$ & $0.17 \pm 0.01$ & $1.15 \pm 0.09$ & $7.77 \pm 0.28$ \\
$(\mathrm{C} 20: 0)$ & $\mathrm{ND}^{*}$ & $0.52 \pm 0.04$ & $0.01 \pm 0.00$ \\
\hline
\end{tabular}

${ }^{*}$ Not detected.

TABLE 2: $R^{2}$ values for PLS model using different spectral frequencies for analysis of red fruit oil (RFO), corn oil (CO), and soybean oil (SO) in ternary mixture.

\begin{tabular}{lccc}
\hline $\begin{array}{l}\text { Frequency } \\
\text { regions }\left(\mathrm{cm}^{-1}\right)\end{array}$ & $\begin{array}{c}R^{2} \text { values of RFO, CO, and SO in ternary mixture } \\
\text { RFO }\end{array}$ & CO & SO \\
\hline $4000-650$ & 0.9793 & 0.8652 & 0.9100 \\
$1500-1800$ & 0.9753 & 0.5532 & 0.5062 \\
$1500-1800$ and & 0.9771 & 0.6689 & 0.7281 \\
$800-1200$ & & & \\
\hline
\end{tabular}

higher $R^{2}$ and lower values of root mean standard error of calibration (RMSEC) were selected; meanwhile, regions that reveal low $R^{2}$ and high RMSEC should be ignored. Based on the highest value of $R^{2}$, frequency region of 4000$650 \mathrm{~cm}^{-1}$ was selected for quantification of RFO, CO, and $\mathrm{SO}$ in ternary mixture systems using PLS calibration. The $R^{2}$ values obtained for RFO, CO, and SO are $0.9793,0.8652$, and 0.9100 , respectively. The full spectral region is widely used because it prevents the overfitting problem by reducing the amount of data in the spectrum without reducing and eliminating one or more useful information.

The optimization process was continued by investigating several treatments of FTIR spectra (normal and derivatives) using selected frequency previously optimized, that is, 4000$650 \mathrm{~cm}^{-1}$. The first derivative removes the common intensity effect of FTIR spectra and can simplify the baseline selection. Meanwhile, the second derivative can eliminate the slope effect [21]. However, derivation treatments can affect the measurement sensitivity. The values of $R^{2}$ and RMSEC were used for calibration criteria, as shown in Table 3. The first derivative spectra were exploited for determination of RFO in ternary mixture with the $R^{2}$ value of 0.9863 and RMSEC value of $1.59 \%(\mathrm{v} / \mathrm{v})$. Furthermore, the levels of CO and SO were better determined using PLS in combination with second derivative spectra with $R^{2}$ values of 0.9276 and 0.9693 and with RMSEC values of 1.72 and $1.60 \%(\mathrm{v} / \mathrm{v})$, respectively. The PLS scatter plot for the relationship between actual value ( $x$-axis) and FTIR predicted value ( $y$-axis) of RFO measured using first derivative FTIR spectra is shown in Figure 2(a). Furthermore, the actual value of $\mathrm{CO}$ and $\mathrm{SO}$ was 


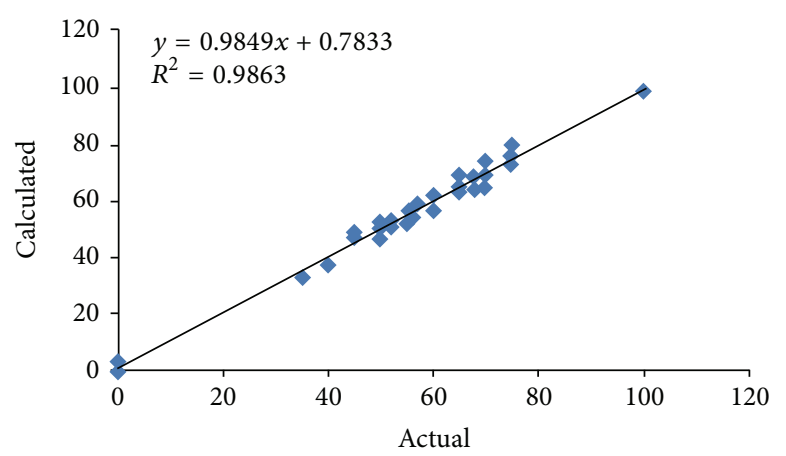

(a)

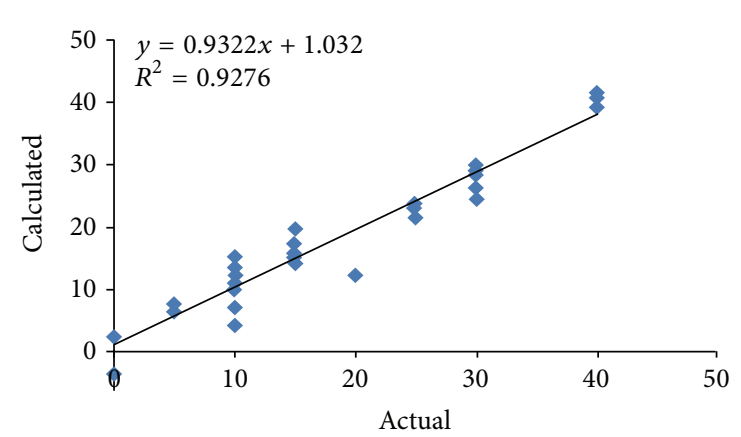

(b)

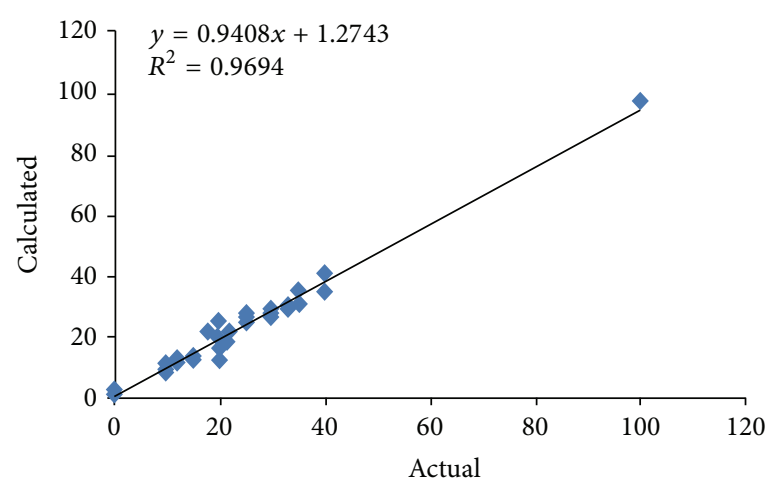

(c)

FIGURE 2: Scatter plot for the relationship between actual value of edible oils and FTIR calculated value. (a) Red fruit oil; (b) corn oil; (c) soybean oil.

TABLE 3: The statistical parameters using PLS calibration at frequency region of $4000-650 \mathrm{~cm}^{-1}$ for determination of red fruit oil (RFO), corn oil (CO), and soybean oil (SO) in ternary mixture systems*.

\begin{tabular}{|c|c|c|c|c|}
\hline \multirow{2}{*}{ Analytes } & \multirow{2}{*}{$\begin{array}{l}\text { Spectral } \\
\text { treatments }\end{array}$} & \multirow{2}{*}{ Factors } & \multicolumn{2}{|c|}{ Calibration performance } \\
\hline & & & $R^{2}$ & RMSEC \\
\hline \multirow{3}{*}{ RFO } & Normal & 3 & 0.9793 & 1.7670 \\
\hline & 1st derivative & 4 & 0.9863 & 1.5877 \\
\hline & 2nd derivative & 5 & 0.9804 & 1.668 \\
\hline \multirow{3}{*}{$\mathrm{CO}$} & Normal & 8 & 0.8652 & 2.0214 \\
\hline & 1st derivative & 2 & 0.0437 & 3.3432 \\
\hline & $2 n d$ derivative & 8 & 0.9276 & 1.7165 \\
\hline \multirow{3}{*}{ SO } & Normal & 9 & 0.9100 & 2.3553 \\
\hline & 1st derivative & 7 & 0.9545 & 1.8015 \\
\hline & 2 nd derivative & 8 & 0.9693 & 1.6007 \\
\hline
\end{tabular}

${ }^{*}$ Spectral treatments selected for quantifications are marked with italics.

also correlated altogether with FTIR predicted values using second derivative spectra, as shown in Figures 2(b) and 2(c).

The calibration model was further validated using independent samples. Table 4 compiled the validation performance of PLS in terms of $R^{2}$ and RMSEP, as well as the equation for the relationship between actual and FTIR predicted values for determination of RFO, $\mathrm{CO}$, and $\mathrm{SO}$ at frequency region of $4000-650 \mathrm{~cm}^{-1}$ using the optimized FTIR spectral treatments. The low value of RMSEP and the high value of $R^{2}$ indicate that FTIR spectroscopy combined with chemometrics of PLS regression and appropriate spectral treatments is an accurate technique for quantification of $\mathrm{RFO}, \mathrm{CO}$, and $\mathrm{SO}$ in ternary mixture system.

\section{Conclusion}

We concluded that FTIR spectroscopy combined with partial least square regression with first derivative spectra at frequency region of $4000-650 \mathrm{~cm}^{-1}$ can be exploited for quantification of RFO in ternary mixture system with SO and $\mathrm{CO}$; meanwhile, second derivative spectra were used for quantification of $\mathrm{CO}$ and $\mathrm{SO}$ in ternary mixture. The $R^{2}$ values obtained for the relationship between actual and FTIR predicted values of RFO, CO, and SO were $0.9863,0.9276$, and 0.9693 with RMSEC values of $1.59,1.72$, and $1.60 \%(\mathrm{v} / \mathrm{v})$, respectively. The developed method is fast, with no excessive sample preparation, and is not involving the use of reagents or chemicals. 
TABLE 4: The performance of validation model used for prediction of red fruit oil (RFO), corn oil (CO), and soybean oil (SO) in ternary mixture.

\begin{tabular}{lcccc}
\hline Analytes & Spectral treatments & Equation & \multicolumn{2}{c}{ Validation performance } \\
RFO & 1st derivative & $y=0.9908 x+0.8464$ & 0.9404 & 4.2774 \\
CO & 2nd derivative & $y=0.7902 x+4.745$ & 0.9624 & 8.5366 \\
SO & 2nd derivative & $y=0.9996 x-0.0717$ & 0.9749 & 3.9805 \\
\hline
\end{tabular}

\section{Conflict of Interests}

The authors declare that there is no conflict of interests regarding the publication of this paper.

\section{Acknowledgment}

The authors thank The Ministry of National Education, Republic of Indonesia, for financial support during this research via Competitive Grant awarded to Professor Sugeng Riyanto.

\section{References}

[1] A. Rohman, S. Riyanto, N. Yuniarti, W. R. Saputra, R. Utami, and M. Mulatsih, "Antioxidant activity, total phenolic, and total flavaonoid of extracts and fractions of red fruit (Pandanus conoideus Lam)," International Food Research Journal, vol. 17, no. 1, pp. 97-106, 2010.

[2] M. Budi and F. R. Paimin, Red Fruit (Pandanus conoideus L), Penebar Swadaya, Jakarta, Indonesia, 2004.

[3] D. L. García-González, R. Aparicio-Ruiz, and R. Aparicio, "Review article: virgin olive oil-chemical implications on quality and health," European Journal of Lipid Science and Technology, vol. 110, no. 7, pp. 602-607, 2008.

[4] A. Rohman and Y. B. C. Man, "FTIR spectroscopy combined with chemometrics for authentication of cod liver oil," Vibrational Spectroscopy, vol. 55, no. 2, pp. 141-145, 2011.

[5] A. Rohman, Y. B. C. Man, A. Ismail, and P. Hashim, "Application of Fourier transform infrared (FTIR) spectroscopy for determination of virgin coconut oil (VCO) in binary mixtures with palm oil and olive oil," Journal of the American Oil Chemists' Society, vol. 87, no. 6, pp. 601-606, 2010.

[6] L. M. Reid, C. P. O’Donnell, and G. Downey, "Recent technological advances for the determination of food authenticity," Trends in Food Science and Technology, vol. 17, no. 7, pp. 344-353, 2006.

[7] F. R. van de Voort, J. Sedman, and T. Russin, "Lipid analysis by vibrational spectroscopy," European Journal of Lipid Science and Technology, vol. 103, no. 12, pp. 815-840, 2001.

[8] A. A. Christy, S. Kasemsumran, Y. Du, and Y. Ozaki, "The detection and quantification of adulteration in olive oil by nearinfrared spectroscopy and chemometrics," Analytical Sciences, vol. 20, no. 6, pp. 935-940, 2004.

[9] Y. B. C. Man, Z. A. Syahariza, and A. Rohman, "Fourier transform infrared (FTIR) spectroscopy: development, techniques, and application in the analyses of fats and oils," in Fourier Transform Infrared Spectroscopy, O. J. Ress, Ed., pp. 1-36, Nova Science, New York, NY, USA, 2010.

[10] A. Rohman and Y. B. C. Man, "Application of fourier transform infrared spectroscopy for authentication of functional food oils," Applied Spectroscopy Reviews, vol. 47, no. 1, pp. 1-13, 2012.
[11] F. Marini, R. Bucci, I. Ginevro, and A. L. Magrì, "Coupling of IR measurements and multivariate calibration techniques for the determination of enantiomeric excess in pharmaceutical preparations," Chemometrics and Intelligent Laboratory Systems, vol. 97, no. 1, pp. 52-63, 2009.

[12] Z. A. Syahariza, Y. B. C. Man, J. Selamat, and J. Bakar, "Detection of lard adulteration in cake formulation by Fourier transform infrared (FTIR) spectroscopy," Food Chemistry, vol. 92, no. 2, pp. 365-371, 2005.

[13] N. Vlachos, Y. Skopelitis, M. Psaroudaki, V. Konstantinidou, A. Chatzilazarou, and E. Tegou, "Applications of Fourier transform-infrared spectroscopy to edible oils," Analytica Chimica Acta, vol. 573-574, pp. 459-465, 2006.

[14] V. Baeten, J. A. F. Pierna, P. Dardenne, M. Meurens, D. L. García-González, and R. Aparicio-Ruiz, "Detection of the presence of hazelnut oil in olive oil by FT-Raman and FT-MIR spectroscopy," Journal of Agricultural and Food Chemistry, vol. 53, no. 16, pp. 6201-6206, 2005.

[15] A. Rohman and Y. B. C. Man, "Palm oil analysis in adulterated sesame oil using chromatography and FTIR spectroscopy," European Journal of Lipid Science and Technology, vol. 113, no. 4, pp. 522-527, 2011.

[16] A. Rohman and Y. B. C. Man, "Analysis of cod-liver oil adulteration using fourier transform infrared (FTIR) spectroscopy," Journal of the American Oil Chemists' Society, vol. 86, no. 12, pp. 1149-1153, 2009.

[17] M. A. Manaf, Y. B. C. Man, N. S. A. Hamid, A. Ismail, and S. Z. Abidin, "Analysis of adulteration of virgin coconut oil by palm kernel olein using fourier transform infrared spectroscopy," Journal of Food Lipids, vol. 14, no. 2, pp. 111-121, 2007.

[18] A. Rohman, Y. B. C. Man, and S. Riyanto, "Authentication analysis of red fruit (Pandanus conoideus lam) oil using FTIR spectroscopy in combination with chemometrics," Phytochemical Analysis, vol. 22, no. 5, pp. 462-467, 2011.

[19] G. Gurdeniz and B. Ozen, "Detection of adulteration of extravirgin olive oil by chemometric analysis of mid-infrared spectral data," Food Chemistry, vol. 116, no. 2, pp. 519-525, 2009.

[20] I. N. Hayati, Y. B. C. Man, C. P. Tan, and I. N. Aini, "Physicochemical characteristics of soybean oil, palm kernel olein, and their binary blends," International Journal of Food Science and Technology, vol. 44, no. 1, pp. 152-161, 2009.

[21] M. J. Lerma-García, G. Ramis-Ramos, J. M. Herrero-Martínez, and E. F. Simó-Alfonso, "Authentication of extra virgin olive oils by Fourier-transform infrared spectroscopy," Food Chemistry, vol. 118, no. 1, pp. 78-83, 2010. 

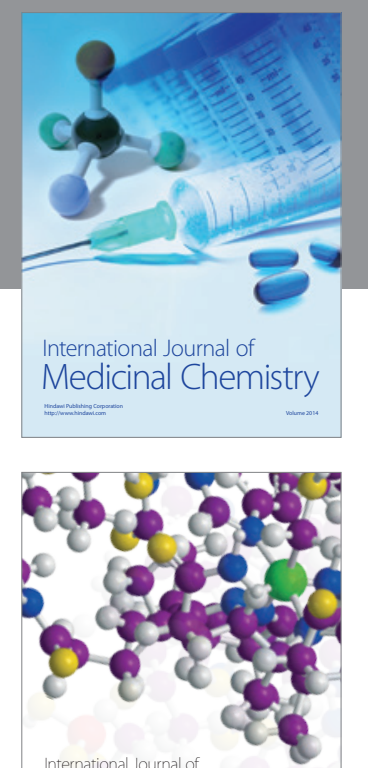

\section{Carbohydrate} Chemistry

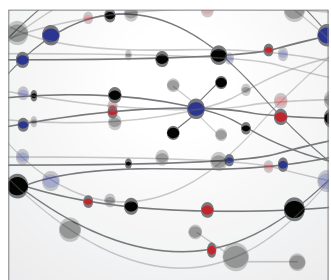

The Scientific World Journal
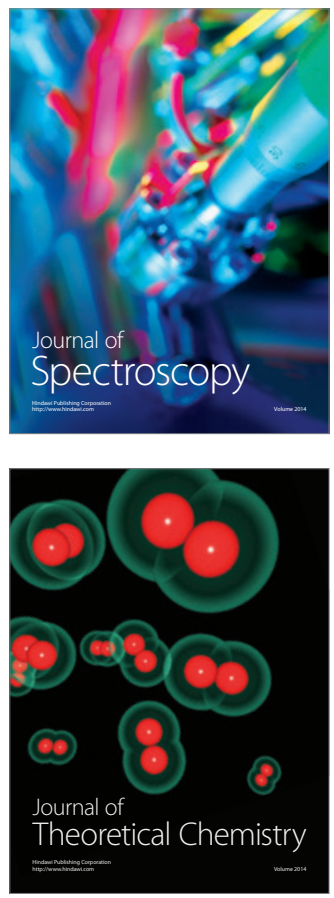
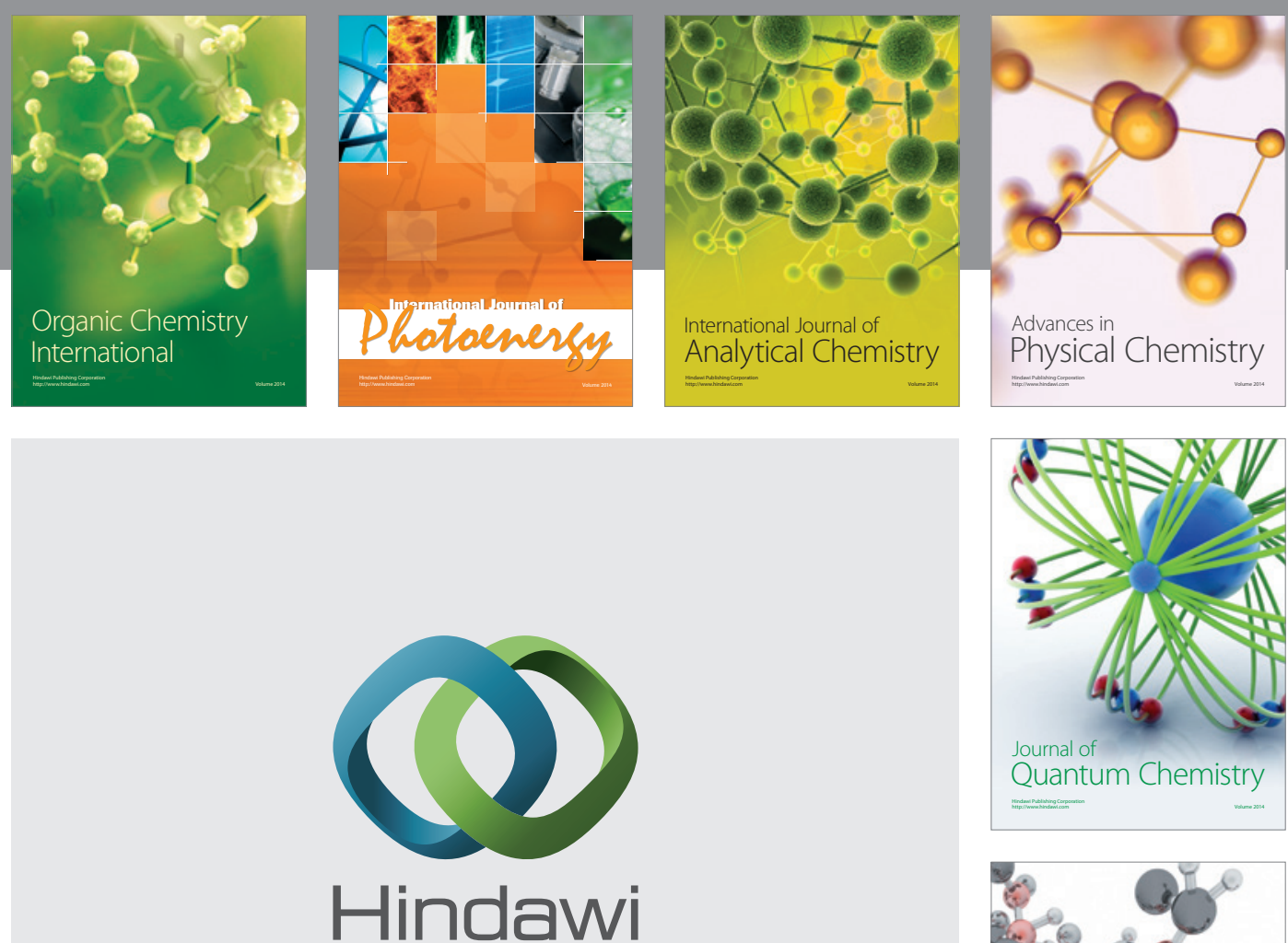

Submit your manuscripts at

http://www.hindawi.com

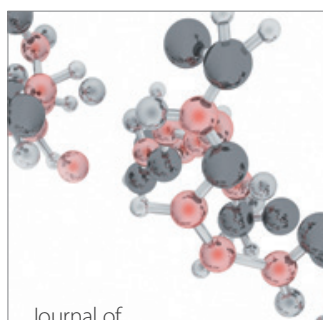

Analytical Methods

in Chemistry

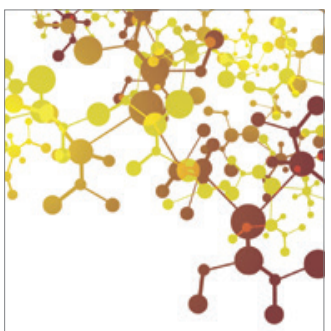

Journal of

Applied Chemistry

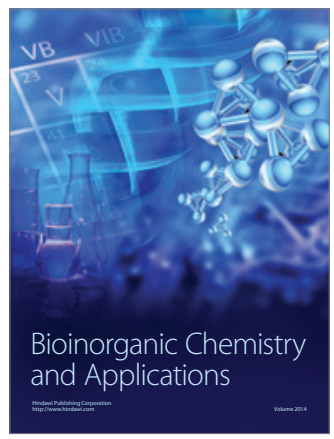

Inorganic Chemistry
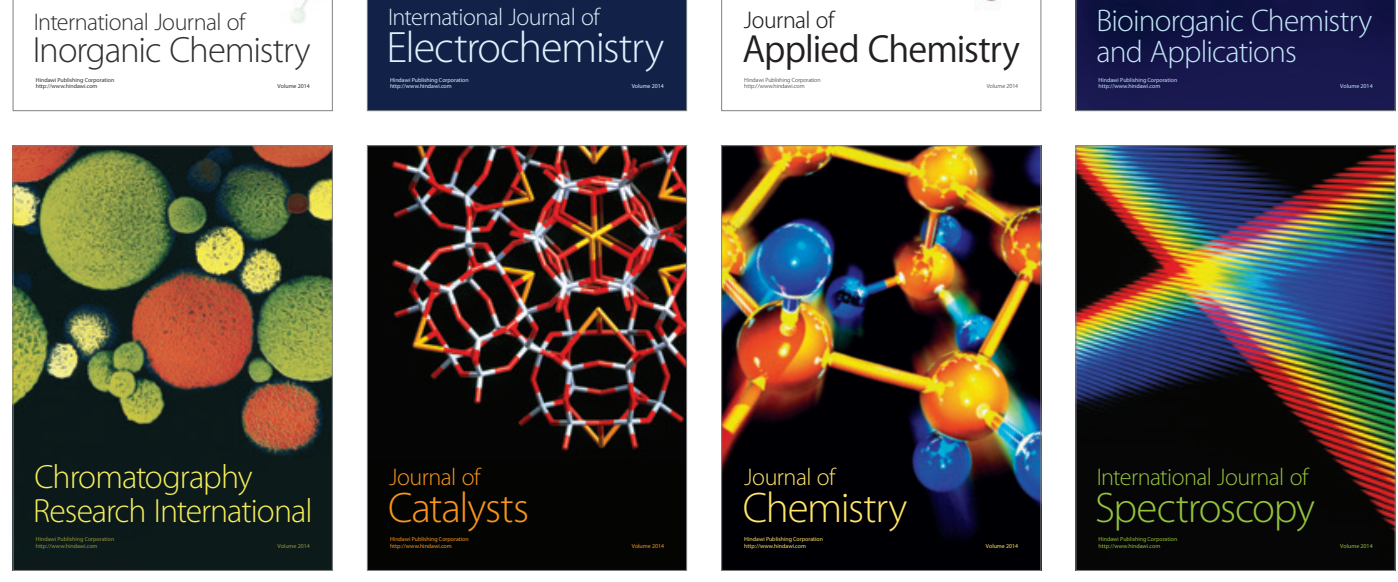